\title{
A DISCRETE AND FINITE APPROACH TO PAST PHYSICAL REALITY
}

\author{
WOLFGANG ORTHUBER
}

Received 25 December 2002

\begin{abstract}
This paper is a synthesis of previously published material on the topic. We show that an adequate mathematical model for the physical (i.e., perceptible and therefore past) reality must be finite. A finite approach to past proper time is given. Proper time turns out to be proportional to the sum of the return probabilities of a Bernoulli random walk.
\end{abstract}

2000 Mathematics Subject Classification: 81P15, 81Q99, 60G50, 83A05, 93C99.

1. Introduction. Every physical measurement needs a finite, different-from-zero measurement time and provides information in the form of the choice of a measurement result from all possible measurement results. If infinitely many (different) measurement results would be possible, the choice of a measurement result could deliver an infinite quantity of information. But the results of physical measurements (of finite duration) never deliver an infinite quantity of information; they describe past, finite reality. Therefore, the set of all possible measurement results is a priori finite. In the physical reality, only a finite information quantity can be processed within a finite time interval. For mathematical models whose representation requires a processing of an infinite quantity of information, for example, irrational numbers, no (exact) equivalent exists in the physical reality. So, mathematical calculations, which have an equivalent in physical reality, can include only rational (finitely many elementary) combinations of rational numbers. Conclusions arise from this for the foundations of mathematical physics.

1.1. On the finite information content of the physically existing reality. In the nineteenth century, it was usual to assume a continuous behavior of physical nature and to use for its description continuous functions with continuous sets as a domain of definition and as a range of values. These sets a priori contain infinitely many elements. Also the axioms of set theory permit the a priori existence of infinite sets and choice functions on those sets. These axioms were formulated in 1900 and led to several paradoxes (antinomies) from the beginning, which led to a discussion on the foundations of mathematics $[8,13,14,38,39,40]$, which also deals with the concept of existence (see below). There were suggestions for different attempts to moderate the difficulties $[4,36,37]$. But a limitation of mathematical liberty remained so that the majority of mathematicians keeps to axioms which demand the a priori existence of infinite sets. This is surely also because of the noteworthy successes of analytical approaches in the description of natural processes. So it is explainable that, in mathematical physics, the 
analytical work with infinite continuous number sets became a not scrutinized selfevident fact (besides exceptions like [16, 17, 18, 19]), despite the mentioned open discussion on the foundations of mathematics and despite the discovery of quantization of physical measurement results at the beginning of the twentieth century. It has been a good opportunity for drawing conclusions with regard to the foundations of mathematical physics, but "the moment was lost" [17, page 15]. We know that a (nonzero) quantity which exists in the physical meaning (which is perceptible) is already past and thus by definition fixed and naturally restricted, that is, it cannot be infinitely small or infinitely large. Concerning the physically existing reality (physical reality), a scientific consensus is possible. Even Hilbert concluded the following result [14, page 165]: "now we have established the finiteness of the reality in two directions: to the infinite small and to the infinite large."

In Section 1, which contains large parts of [31], it is shown that the finite information quantity of every measurement result is closely connected with the quantization and even with the finiteness of the set of all possible measurement results. So, for continuous number sets, no equivalent can exist in physical reality.

\subsection{Finite information from physical measurement results}

1.2.1. Information from choices within sets. Sets can be created by the subdivision of a totality into several components or elements. During the creation of a set, the choice of a sequence of elements or subsets is possible. Both the choice and the order of choices contain information. Every perception or every physical measurement provides information in the form of the choice of a measurement result from all possible measurement results.

1.2.2. Quantum physical aspects. The quantum mechanical discoveries at the beginning of the twentieth century have shown reductions of measuring precision as a matter of principle. Location and impulse of a particle, for example, are never simultaneously measurable with arbitrary precision. In the end, this is a consequence of the effect quantization, that is, the fact that only the effect differences are measurable, which are multiples of the half effect quantum $\hbar / 2$.

REASONS FOR CONTINUOUS APPROACHES. For a long time, this quantization has been undiscovered because such small effect differences are not relevant in the case of usual macroscopic measurements: the systems to be measured are often in a complex way composed of many parts whose mutual interaction and whose interaction with the surroundings are not exactly known. For that reason, there are many possibilities of uncontrolled influence on the measurement result so that its variance is so great that effect differences in the order of $\hbar$ have no significant influence on the measurement result. Therefore, in case of macroscopic considerations, it is justified to assume $\hbar$ as negligibly small and to use analytical concepts.

FINITE INFORMATION FROM MEASUREMENT RESULTS. In atomic and subatomic physics, the quantization of the effect becomes evident [26, page 47] and it is also very important from the information-theoretic point of view. Every information transmission means the transfer of free energy from a transmitter to a receiver. In case of 
a physical measurement, the receiver consists of one or several sensors of the measurement equipment. (If the absorption of energy at an object should be measured, the measurement is done indirectly: initially the measurement equipment sends out free energy which, after interaction with the object, is received again by sensors of the measurement equipment, from which the measurement information results.)

During measuring, energy is transferred by photons to rest mass in the sensors. The more energy is necessary, the shorter the measurement time is. If the measurement time is $t_{m}<\infty$, then every photon at least transfers the energy $\hbar / t_{m}$. Since the available free energy is finite, only a finite number of photons are transferable to the sensors within the measurement time. Energy quantities whose difference is less than $\hbar / t_{m}$, in principle, are not distinguishable [26, page 129], that is, every photon has only finitely many distinguishable possibilities for influencing the measurement equipment, respectively, the measurement result. Due to the finite number of sensors in which photons are absorbed, only a finite number of measurement results are possible. It is well known that this restriction is a matter of principle; it is also valid in case of an ideal, maximal exact measurement. So, every measurement result is a choice from an a priori finite set and so it has only finite information content. Of course, another statement would contradict any everyday experience which shows that the complete information of all measurement results known by us is finite, it corresponds to the finite information quantity which can be known by us up to some fixed time.

For clarification, the reasoning now will be specified more precisely by informationtheoretic argumentation. Readers who are familiar with the possible pathologies of continuous probability distributions may skip Section 1.2.3 and the beginning of Section 1.2.4, and continue with Section 1.3.

1.2.3. Information and entropy. Every measurement is an experiment whose result is the measurement result. Let $H(\beta)$ denote the entropy of an experiment $\beta$, which quantifies its uncertainty (the introduced entropy concept is closely connected to the one of thermodynamics, cf. [32] and especially [5]). If $J$ is a set of indices, $M:=\left\{A_{k}: k \in J\right\}$ is the set of all results of the experiment $\beta$, and $p\left(A_{k}\right)$ are their probabilities, then the entropy $H(\beta)$ is defined by

$$
H(\beta):=-\sum_{k \in J} p\left(A_{k}\right) \log _{2} p\left(A_{k}\right)
$$

(cf. [15, page 59]). The entropy $H(\beta)$ is nonnegative because $\log _{2} p\left(A_{k}\right) \leq 0$. If $H(\beta)=0$, the result of the experiment $\beta$ is known in advance. A larger or smaller value of $H(\beta)$ corresponds to a larger or smaller uncertainty of the result. Now, let $\alpha$ be an experiment which precedes $\beta$. The result of $\alpha$ can limit the number of possible results of $\beta$ and so it reduces its uncertainty, respectively, the entropy $H(\beta)$. The entropy of $\beta$ after execution of $\alpha$ is called conditional entropy and we write it as $H_{\alpha}(\beta)$. If $\beta$ is independent of $\alpha$, the realization of $\alpha$ does not reduce the entropy of $\beta$, that is, $H_{\alpha}(\beta)=H(\beta)$. If the result of $\alpha$ completely determines the result of $\beta$, the conditional entropy $H_{\alpha}(\beta)$ is zero. The difference

$$
I(\alpha, \beta):=H(\beta)-H_{\alpha}(\beta)
$$


is called the quantity of information contained in the result of $\alpha$ about the result of $\beta$, or in short the information contained in $\alpha$ about $\beta$ (cf. [15, page 86]). It shows how much the realization of $\alpha$ reduces the uncertainty of $\beta$ and how much we learn from the result of $\alpha$ about the result of $\beta$.

ENTROPY OF PHYSICAL EXPERIMENTS. Usually, the result of a physical experiment is represented by a vector (if necessary, a multidimensional vector), whose components are real numbers. Because the real numbers form a continuous ordered set, which (equipped with a metric) is a Hausdorff space, such representation implies infinitely many different possibilities for the result of the experiment. So, in (1.1), the defined entropy cannot have a finite value [15, page 92].

Without restriction of generality, we clarify this by an example of a physical experiment $\beta$, whose result is a one-dimensional quantity which is represented by a real number $x \geq 0$ (multiplied by a unit), for example, a length. We assume that $x$ is finite, that is, there is a number $s$ so that $s>x \geq 0$ holds. For a given set $M \subset \mathbb{R}$, we write $p(M)$ for the probability that the result is contained in $M$.

We now suppose a continuous probability distribution of possible results within the interval $[0, s$. We can always find two numbers $a, b \in[0, s[$ with $a<b$ and $1 / e>$ $p([a, b[)>0$. The interval $[a, b[\subset[0, s[$ can be so small that the probability is distributed nearly equally within it. Then we can assume that for all $n \in \mathbb{N} \backslash\{0\}$ and $k \in\{1, \ldots, n\}$ the probability for the intervals

$$
J_{k}:=\left[a+(k-1) \frac{b-a}{n}, a+k \frac{b-a}{n}[\right.
$$

is nearly equal, that is, $p\left(J_{k}\right) \approx p([a, b[) / n$, and with $\epsilon:=p([a, b[) / 2$, particularly,

$$
0<\frac{\epsilon}{n}=\frac{p([a, b[)}{2 n}<p\left(J_{k}\right)
$$

holds. The function $f:] 0, \infty\left[\rightarrow \mathbb{R}, x \rightarrow-x \log _{2} x\right.$, is strictly increasing in $] 0,1 / e[$ and $\left.p\left(J_{k}\right) \in\right] 0,1 / e[$. From this and (1.1), for the entropy $H(\beta)$ of the experiment, it follows that

$$
\begin{aligned}
H(\beta) & \geq \sum_{k=1}^{n}\left(-p\left(J_{k}\right) \log _{2} p\left(J_{k}\right)\right) \\
& >\sum_{k=1}^{n}\left(-\frac{\epsilon}{n} \log _{2} \frac{\epsilon}{n}\right)=-\epsilon \log _{2} \frac{\epsilon}{n}=\epsilon\left(\log _{2} n-\log _{2} \epsilon\right) .
\end{aligned}
$$

Since $n$ can be arbitrarily large, we cannot get a finite value for the entropy $H(\beta)$ of the experiment $\beta$. Such situation always arises if we start out the assumption that a continuous set of numbers represents the set of possible results of an experiment (cf. also [15, page 93]). After execution of $\beta$, a number (the measurement result) $x \in[0, s$ [ has probability 1 , while all other numbers have probability 0 , so that the conditional 
entropy $H_{\beta}(\beta)$ of $\beta$ is $H_{\beta}(\beta)=p(x) \log _{2} p(x)=1 \log _{2} 1=0$. Insertion of this into (1.2) gives the information quantity which we receive from the execution of the experiment $\beta$ :

$$
I(\beta, \beta)=H(\beta)-H_{\beta}(\beta)=H(\beta)-0=H(\beta) .
$$

From this and (1.5), it follows that both $H(\beta)$ and $I(\beta, \beta)$ are not finite. In a nutshell, the measurement result (of the experiment $\beta$ ) has infinite information quantity.

But all experiences from (finite) past have shown that measurement results (results of experiments with finite duration) always have only finite information quantity.

1.2.4. Finite information and finite measuring accuracy. Usually, one says that the reason for finite information of experimental results is finite measuring accuracy (which can also be a matter of principle because of quantum physical reasons, resp., indetermination).

A physically possible experiment (which is always feasible within finite time) is not the above-mentioned experiment $\beta$ whose result is a number $x \in[0, s[$, but is at best an experiment $\alpha$ with finite measuring accuracy $\delta>0$ whose result is an interval $\left[x_{\alpha}-\right.$ $\delta, x_{\alpha}+\delta[\subset[0, s]$, so that the result $x$ of the experiment $\beta$ lies within this interval with great probability. After using some simplifications, it can be shown that the result of $\alpha$ contains only finite information (cf. [15, page 92]), that is, the experiment $\alpha$ is physically possible.

THE CONCEPT OF "MEASURING ACCURACY" MUST HAVE A BASIS. The problem of this reasoning is the usage of the term result $x$ of the experiment $\beta$. This $x \in[0, s[$ is the result of an experiment which is not physically feasible, not even in the potential sense. Terms which never have an equivalent in physical reality are used. So the basis for the argumentation is missing.

This problem always occurs when one speaks about an experimental result represented by a selection from an infinite set of possible results, for instance, in the form of a number from a continuum. In this case, the entropy and the gain of information (1.6) are not finite. So the experiment is not feasible within finite time, that is, it is not physically possible. Therefore, the conclusion in Section 1.2.2 can be found also in purely information theoretical way. We must consider that also the duration of the experiment contains information, and so there are also only finitely many possibilities for the duration.

1.3. The finiteness of the set of possible measurement results. We summarize the above results in the following theorem.

THEOREM 1.1. Let $\beta$ denote a physical experiment (which is completed after finite time). Then there are only finitely many possibilities for the duration and for the results of $\beta$. Each result represents the choice from an a priori only finite set of possible results.

From this we can easily deduce useful conclusions for physical calculations.

1.3.1. Indexing experimental results. For instance, an index (if necessary, multidimensional) over all possible experimental results is possible and the sequence of 
the index is freely selectable (among others due to topological criteria or informationtheoretic coding depth). The simplest possibility is a one-dimensional index. If $M$ is the set of all possible (different) results of a physical experiment (an experiment of finite duration), we can write $M$ in the form $M=\left\{y_{1}, y_{2}, \ldots, y_{|M|}\right\}$, in which $y_{k}$ can be vectors which, respectively, represent an experimental result.

EXAMPLE OF A SYMMETRICAL INDEX. It is often useful to consider symmetries. One can choose the index symmetrically to a single experimental result or a couple of experimental results and represent the set $M$ of all possible experimental results $y_{k}$ in the following form:

$$
M=\left\{y_{-|M|+1}, y_{-|M|+3}, \ldots, y_{-2}, y_{0}, y_{2}, \ldots, y_{|M|-3}, y_{|M|-1}\right\}
$$

if $|M|$ is odd, and

$$
M=\left\{y_{-|M|+1}, y_{-|M|+3}, \ldots, y_{-1}, y_{1}, \ldots, y_{|M|-3}, y_{|M|-1}\right\}
$$

if $|M|$ is even.

1.3.2. Finiteness of realistic physical calculations. If an estimation of possible results of a physical experiment should be given, one has to consider that the information quantity of both the initial data and every possible experimental result is finite. So, with the help of a mathematical model from the initial data, a probability distribution over a finite set of possible experimental results has to be calculated. Particularly, each experimental result, respectively, each equivalent result of a calculation, contains only finite information. So there is a possibility to calculate the result exactly from the initial data using only a finite number of elementary steps. We specify this now in a more precise way.

DEFINITION 1.2 (elementary combination). All permitted combinations of rational numbers by one of the four basic arithmetic operations (i.e., addition, subtraction, multiplication, division) are called elementary combinations.

So, for $a, b \in \mathbb{Q}$, there are exactly the elementary combinations $a+b=b+a, a-b, b-$ $a, a b=b a, a / b, b / a$; in the last two cases, $b \neq 0, a \neq 0$, respectively, are presupposed. We know that for each elementary combination within finite time an exact equivalent can exist in the physical reality (e.g., in the form of a finite sequence of binary decisions).

Chaining ElementARy Combinations. Now, for $n \in \mathbb{N}, a \in \mathbb{Q} \backslash\{0\}$, we denote by $M_{n}(a)$ the set of all numbers which can be formed from $a$ by chaining $n$ elementary combinations. If $n \in \mathbb{N}$ is a predefined (finite) number, then $\left|M_{n}(a)\right|$ is finite. In the reverse case, if $n$ is selectable subsequently and arbitrary large, there is no upper bound for $\left|M_{n}(a)\right|$. The initial data of a physical experiment (of finite duration) represent the choice from a finite number of possible initial data (because of their finite information content); likewise, the end data and the experimental result, respectively. If the initial data are represented as numbers which are not all equal to 0 , we can get an infinite number of possible results if we can combine them by infinitely many elementary 
combinations. But, a priori, we know that in case of a physical experiment (i.e., after predefined maximal time for the experiment), only a finite number of different possibilities of experimental results and only a finite number of equivalent arithmetical results are possible. So, for a mathematical calculation which is conformal to physical reality, there is an upper bound $\tilde{n} \in \mathbb{N}$ for the count $n$ of used elementary combinations to get the result. Particularly, all numbers representing experimental results are values of rational functions of the initial data. Since the initial data are also results of experiments with finite duration, we can start out the assumption that the numbers which represent these data are rational if their units are chosen in a simple way, which we will assume subsequently. (This means that the definition is done without analytical models and no irrational number factors are contained in them. We, otherwise, have to admit numbers from a finite field extension of $\mathbb{Q}$.)

So, mathematical calculations which have an equivalent in physical reality can include only rational (finitely many elementary) combinations of rational numbers. At this, we know, because of quantum physical results, that, as a rule, the end data are not determined by the initial data, that is, they do not contain enough information for determination. So, the result of the calculation will be a probability distribution of possible results. Each of them is calculated from the initial data by a finite sequence of elementary combinations. The choice of a certain sequence is done during the experiment by a finite number of decisions so that the probability for a certain sequence is also a rational number. We summarize this in the following theorem.

THEOREM 1.3. Let $x \in \mathbb{Q}^{l}$ denote the l-dimensional vector of the initial data of a physical experiment (with a given finite duration). There are only finitely many different possibilities for the experimental result. If $y_{j}, j \in\{1,2, \ldots, n\}$, are the possible $m$-dimensional result vectors with the probabilities $p_{j}$, both $y_{j}$ and $p_{j}$ result from $x$ by a finite number of elementary combinations. Particularly, they are results of rational functions of $x$.

So, one way to a better understanding of the physical nature is the study of rational functions; for example, finite partial sums of power series whose results lie close to the results of analytical functions which are frequently used in the mathematical physics. The next section shows an example of this.

2. A discrete and finite approach to past proper time. The function $\gamma(x)=1 / \sqrt{1-x^{2}}$ plays an important role in the mathematical physics, for example, as a factor for relativistic time dilation in case of $x=\beta$ with $\beta=v / c$ or $\beta=p c / E$. Due to the above considerations, it is reasonable to study the power series expansion of $\gamma(x)$. In this section, its relationship with the binomial distribution is shown, especially the fact that the summands of the power series correspond to the return probabilities to the starting point (local coordinates, configuration, or state) of a Bernoulli random walk. So, $\gamma(x)$, and with that also proper time, is proportional to the sum of the return probabilities of a Bernoulli random walk. In case of $x=1$ or $v=c$, the random walk is symmetric. Random walks with absorbing barriers are introduced in the appendix. In Section 2, which contains large parts of [29], essentially the basic mathematical facts are shown and references are given, most interpretation is left to the reader. 
2.1. Motivation. From Section 1, we know that the (measurable) result data vector of a physical experiment (with finite duration) can be calculated from the (measurable) initial data vector by combining a finite number of basic arithmetic operations. This does not contradict the fact that many analytical functions with infinite power series expansions can successfully predict (approximative) experimental results: they are only successful in the case of convergence, that is, in the case of convergence of the partial sum sequence of the corresponding power series expansion. We can choose an arbitrary long but finite partial sum, calculating it by finitely many basic arithmetic operations and the result is arbitrarily near to the value of the infinite power series (which is the value of the corresponding function). So there can always be an exact partial sum and an approximative function result without a chance for experimental distinction. However, the study of partial sums is the possibility to learn more about the nature of the underlying (finite) physical process-even in the case of missing convergence. Here, we study the function

$$
\gamma:]-1,1\left[\rightarrow \mathbb{R}, \quad \gamma(x)=\frac{1}{\sqrt{1-x^{2}}},\right.
$$

which is frequently used in the mathematical physics, for example, as a factor for relativistic time dilation in case of $x=\beta$ with $\beta=v / c$ or $\beta=p c / E$. We investigate the power series representation of $\gamma(x)$ and show its relationship with the binomial distribution, which plays an important role in nature, often in a complex way, compare $[1,2,3,9,10,12,21,22,25,27,28,33,35]$. Recall the close connection between relativistic mass increase and time dilation, especially when reading [3], in which concrete physical relevance of finite partial sums (of the power series expansion of $1 / \sqrt{1-\hat{x}}$ ) is shown. In the appendix, we also consider $1 / \gamma(x)$.

\subsection{The connection of proper time and return probabilities}

2.2.1. The binomial series. In case $\alpha \in \mathbb{Z}^{*}=\{j \in \mathbb{Z} \mid j \geq 0\}$, the function

$$
\hat{f}_{\alpha}: \mathbb{C} \longrightarrow \mathbb{C}, \quad \hat{f}_{\alpha}(z)=(1+z)^{\alpha},
$$

has a finite power series expansion of the form

$$
\hat{f}_{\alpha}(z)=1+\left(\begin{array}{l}
\alpha \\
1
\end{array}\right) z^{l}+\left(\begin{array}{l}
\alpha \\
2
\end{array}\right) z^{2}+\cdots+\left(\begin{array}{c}
\alpha \\
\alpha
\end{array}\right) z^{\alpha}=\sum_{l=0}^{\alpha}\left(\begin{array}{l}
\alpha \\
l
\end{array}\right) z^{l}
$$

in which $\left(\begin{array}{l}\alpha \\ l\end{array}\right)$ are the binomial coefficients which are defined by

$$
\left(\begin{array}{l}
\alpha \\
0
\end{array}\right)=1, \quad\left(\begin{array}{l}
\alpha \\
l
\end{array}\right)=\frac{\alpha(\alpha-1)(\alpha-2) \cdots(\alpha-l+1)}{l !} \text { for } l \in \mathbb{Z}^{*} \backslash\{0\} .
$$

In case $\alpha \in \mathbb{C} \backslash \mathbb{Z}^{*}$ and $|z|<1$, we can develop the function $\hat{f}_{\alpha}(z)=(1+z)^{\alpha}$ into a convergent MacLaurin series $[11,20,23,24]$. If $f_{\alpha}(z)$ denotes the principal value of $\hat{f}_{\alpha}(z)$, which is equal to 1 at $z=0$, we obtain

$$
f_{\alpha}(0)=1, \quad f_{\alpha}^{\prime}(0)=\alpha, \quad f_{\alpha}^{\prime \prime}(0)=\alpha(\alpha-1), \ldots, f_{\alpha}^{(l)}=\left(\begin{array}{c}
\alpha \\
l
\end{array}\right) l !,
$$


from which the representation of $f_{\alpha}(z)$ as binomial series: follows

$$
f_{\alpha}(z)=\sum_{l=0}^{\infty}\left(\begin{array}{l}
\alpha \\
l
\end{array}\right) z^{l}
$$

2.2.2. The power series of $\gamma(x)=1 / \sqrt{1-x^{2}}$. Since $1 / \sqrt{1+z}=f_{-1 / 2}(z)$, we get with (2.4) and (2.6),

$$
\begin{aligned}
\frac{1}{\sqrt{1+z}}= & \sum_{l=0}^{\infty}\left(\begin{array}{c}
-\frac{1}{2} \\
l
\end{array}\right) z^{l} \\
= & 1+\frac{-1 / 2}{1} z^{1}+\frac{(-1 / 2) \cdot(-3 / 2)}{1 \cdot 2} z^{2}+\frac{(-1 / 2) \cdot(-3 / 2) \cdot(-5 / 2)}{1 \cdot 2 \cdot 3} z^{3} \\
& +\frac{(-1 / 2) \cdot(-3 / 2) \cdot(-5 / 2) \cdot(-7 / 2)}{1 \cdot 2 \cdot 3 \cdot 4} z^{4}+\cdots \\
= & 1-\frac{1}{2^{1} \cdot 1 !} z^{1}+\frac{1 \cdot 3}{2^{2} \cdot 2 !} z^{2}-\frac{1 \cdot 3 \cdot 5}{2^{3} \cdot 3 !} z^{3}+\frac{1 \cdot 3 \cdot 5 \cdot 7}{2^{4} \cdot 4 !} z^{4}-\cdots \\
= & 1-\frac{2 !}{\left(2^{1} \cdot 1 !\right)^{2}} z^{1}+\frac{4 !}{\left(2^{2} \cdot 2 !\right)^{2}} z^{2}-\frac{6 !}{\left(2^{3} \cdot 3 !\right)^{2}} z^{3}+\frac{8 !}{\left(2^{4} \cdot 4 !\right)^{2}} z^{4}-\cdots \\
= & \sum_{l=0}^{\infty}(-1)^{l} \frac{(2 l) !}{\left(2^{l} \cdot l !\right)^{2}} z^{l} \\
= & \sum_{l=0}^{\infty}\left(\begin{array}{c}
2 l \\
l
\end{array}\right)\left(-\frac{z}{4}\right)^{l},
\end{aligned}
$$

and after the substitution of $z$ by $-x^{2}$,

$$
\gamma(x)=\frac{1}{\sqrt{1-x^{2}}}=\sum_{l=0}^{\infty}\left(\begin{array}{c}
2 l \\
l
\end{array}\right)\left(\frac{x}{2}\right)^{2 l} .
$$

2.2.3. Bernoulli random walk. A Bernoulli random walk is a stochastic process generated by a sequence of Bernoulli trials, that is, independent trials, each of which can have only two results; for example, "positive" (with probability $p$ ) or "negative" (with probability $1-p$ ) $[6,7,34]$. It can be interpreted as a model for the movement of a particle in a one-dimensional discrete state space and may be described in the following terms: the particle moves "randomly" along a line over a lattice of equidistant points (states), which are indexed by an integer coordinate $k$. With every trial, the particle makes a step from point $k$ to point $k+1$ with a given probability $p$ (positive direction) or a step from point $k$ to point $k-1$ with a probability $1-p$ (negative direction).

For $n \in\{1,2,3, \ldots\}$, we denote by $Q O P(n, k, p)$ the probability that the particle is at point $k$ after the $n$th step, and by $Q O P(0, k, p)$ the same probability but before the first step. We assume the starting point of the movement at $k=0$, so $Q O P(0,0, p)=1$ and $Q O P(0, k, p)=0$ for $k \neq 0$, and furthermore,

$$
Q O P(n+1, k, p)=p Q O P(n, k-1, p)+(1-p) Q O P(n, k+1, p) .
$$


When making $n$ trials, only the point $k$ is within reach if $n-k$ and $n+k$ are nonnegative even numbers. We will presuppose this subsequently. There are exactly $\left(\begin{array}{c}n \\ (n+k / 2)\end{array}\right)$ ways with $n+k / 2$ steps in positive direction and $n-k / 2$ steps in negative direction, which lead to point $k$ after the $n$th step. They, respectively, have the probability ( $1-$ $p)^{(n-k) / 2} p^{(n+k) / 2}$. So the chaining of these Bernoulli trials results in the binomial distribution

$$
Q 0 P(n, k, p)=\left(\begin{array}{c}
n \\
\frac{n+k}{2}
\end{array}\right) p^{(n+k) / 2}(1-p)^{(n-k) / 2} .
$$

We now look at the probabilities of returning to the starting point. Because the movement started at $k=0$, these probabilities correspond to

$$
Q O P(2 n, 0, p)=\left(\begin{array}{c}
2 n \\
n
\end{array}\right)(1-p)^{n} p^{n},
$$

that is, $Q O P(2 n, 0, p)$ is the return probability after the $2 n$th step (return is only possible after an even number of steps). Substitution of $p$ by $\left(1-\sqrt{1-x^{2}}\right) / 2$ or $(1+$ $\left.\sqrt{1-x^{2}}\right) / 2$ yields

$$
\begin{aligned}
Q 0 P\left(2 n, 0, \frac{1-\sqrt{1-x^{2}}}{2}\right) & =Q 0 P\left(2 n, 0, \frac{1+\sqrt{1-x^{2}}}{2}\right) \\
& =\left(\begin{array}{c}
2 n \\
n
\end{array}\right)\left(\frac{1-\sqrt{1-x^{2}}}{2} \frac{1+\sqrt{1-x^{2}}}{2}\right)^{n} \\
& =\left(\begin{array}{c}
2 n \\
n
\end{array}\right)\left(\frac{x^{2}}{4}\right)^{n}=\left(\begin{array}{c}
2 n \\
n
\end{array}\right)\left(\frac{x}{2}\right)^{2 n},
\end{aligned}
$$

and by (2.8), we obtain

$$
\begin{aligned}
\gamma(x) & =\frac{1}{\sqrt{1-x^{2}}}=\sum_{n=0}^{\infty} Q O P\left(2 n, 0, \frac{1-\sqrt{1-x^{2}}}{2}\right) \\
& =\sum_{n=0}^{\infty} Q O P\left(2 n, 0, \frac{1+\sqrt{1-x^{2}}}{2}\right) .
\end{aligned}
$$

Note that the condition

$$
p \in\left\{\frac{1-\sqrt{1-x^{2}}}{2}, \frac{1+\sqrt{1-x^{2}}}{2}\right\}
$$

is equivalent to

$$
4 p(1-p)=x^{2}
$$

Before we continue, we should remember that the function $\gamma(x)$ cannot have an exact equivalent in physical (past) reality because the sums in (2.13) are not finite. Furthermore, the values $Q O P(2 n, 0, p)$ are probabilities, and every expectation value 
calculated from a probability has only an average, approximative meaning. Therefore, we presuppose that the random walk contains a sufficiently large number of steps, so that there can be an equivalent to finite partial sums of both sums in (2.13) sufficiently close to $\gamma(x)$, so that the reliability of the expectation value calculated from it is so great that the difference between the individual (discrete) measurement result and the calculated value is not significant.

We can summarize the proportionality of proper time to the sum of the return probabilities in the following theorem.

THEOREM 2.1. Let $\gamma(x)=1 / \sqrt{1-x^{2}}$ represent the (approximative) time dilation factor of reference system $A$ relative to reference system $B$ (it can be assumed that $x=v / c$ if $B$ is moving with velocity $v$ relative to $A$ in flat space-time). Then proper time of $A$ relative to $B$ is (approximatively) proportional to the sum of the return probabilities to the starting point of a Bernoulli random walk, in which each step is directed from point $k$ to $k+1$ with probability $p$ and from point $k$ to $k-1$ with probability $1-p$. At this $4 p(1-p)=x^{2}$ holds.

Every point can represent a state in a one-dimensional discrete state space and $k$ can represent the integer index to it. The reversal of the order of the index is possible and has the same effect as exchanging the probabilities $p$ and $1-p$.

2.2.4. Case $x=1$, respectively, $v=c$. In many physical situations, $x=1$, especially if $x=v / c$ and $v=c$, is the velocity of light, respectively, photons. (Because $v=c$ is the maximal speed of information transport, this case is also important from the information-theoretic point of view). So the case $x=1$ is extremely frequent. We are now able to give an explanation for this.

Equation (2.15) shows that $x=1$ corresponds to $p=1-p=1 / 2$, that is, the probabilities $p$ and $1-p$ of positive and negative step directions are equal. Now, the reason for $x=1$, respectively, $v=c$, for photons becomes clear. Only in case $v=c$, both directions have the same chance. Nature a priori makes no preferences.

SYMMETRIC RANDOM WALK. In case $x=1$, because $p=1-p=1 / 2$, the random walk is symmetric. The accompanying probabilities are

$$
Q 0(n, k):=Q O P\left(n, k, \frac{1}{2}\right)=\left(\begin{array}{c}
n \\
\frac{n+k}{2}
\end{array}\right)\left(\frac{1}{2}\right)^{n} \text {. }
$$

The first values of $Q 0(n, k)$ are shown in Table 2.1.

FINITE RANDOM WALK-FINITE PARTIAL SUM $\gamma_{2 n}(x)$ OF $\gamma(x)$. Now we again consider $\gamma(x)$. In case $x=1$, the series (2.13) does not converge, that is, the infinite sum does not even have an approximative result. But, anyway, we know that an infinite sum cannot have an equivalent in physical reality. So, it is only consequent to consider the finite partial sums

$$
\gamma_{2 n}(x):=\sum_{m=0}^{n} Q O P\left(2 m, 0, \frac{1+\sqrt{1-x^{2}}}{2}\right)
$$


TABLE 2.1. The first values of $Q 0(n, k)$. The representation is chosen in a way that the well-known Pascal triangle gets visible. The number in row $n$ and column $k$ represents the number of ways which lead to point $k$ after the $n$th step. Multiplication by the factor $2^{-n}$ yields $Q 0(n, k)$. The last column contains these factors. In every row $n$ the number of ways which lead back to the origin after $n$ steps is underlined. Multiplication by $2^{-n}$ yields the return probability $Q 0(n, 0)$.

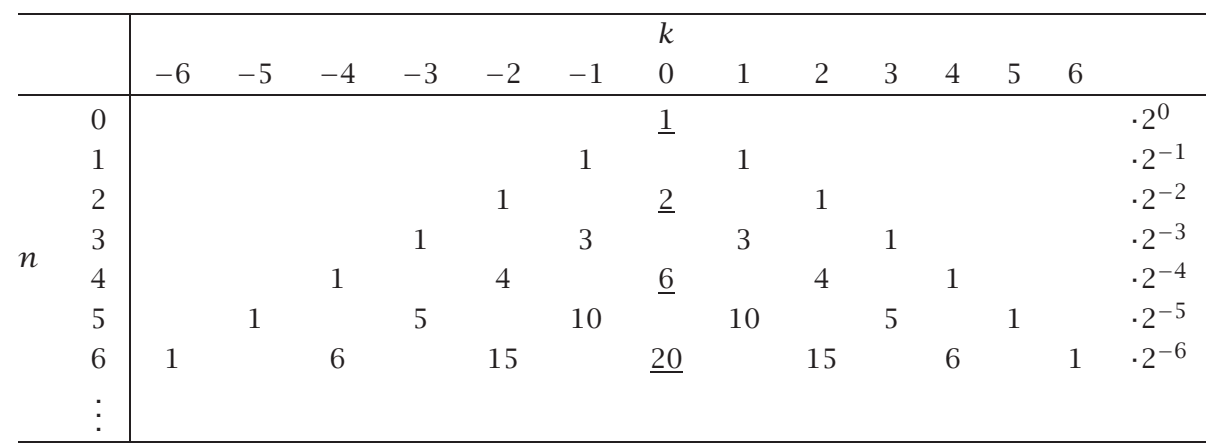

of (2.13). By definition $\gamma_{\infty}(x)=\gamma(x)$, additionally for every (finite) integer $n$,

$$
\gamma_{2 n}(1)=\gamma_{n}(-1)=\sum_{m=0}^{n} Q O P\left(2 m, 0, \frac{1}{2}\right)=\sum_{m=0}^{n} Q 0(2 m, 0)
$$

also exists. It is not difficult to find a closed form for the last sum in (2.18). From

$$
\begin{aligned}
(2 n- & 1) Q 0(2 n-2,0)+Q 0(2 n, 0) \\
& =(2 n-1) \frac{(2 n-2) !}{2^{2 n-2}(n-1) !^{2}}+\frac{(2 n) !}{2^{2 n} n !^{2}} \\
& =\frac{2 n(2 n) !}{(2 n)^{2} 2^{2 n-2}(n-1) !^{2}}+\frac{(2 n) !}{2^{2 n} n !^{2}} \\
& =\frac{2 n(2 n) !}{n^{2} 2^{2 n}(n-1) !^{2}}+\frac{(2 n) !}{2^{2 n} n !^{2}} \\
& =\frac{2 n(2 n) !}{2^{2 n} n !^{2}}+\frac{(2 n) !}{2^{2 n} n !^{2}} \\
& =(2 n+1) \frac{(2 n) !}{2^{2 n} n !^{2}} \\
& =(2 n+1) Q 0(2 n, 0),
\end{aligned}
$$

it follows, by induction, that

$$
\sum_{m=0}^{n} Q 0(2 m, 0)=(2 n+1) Q 0(2 n, 0),
$$


and by (2.18),

$$
\begin{aligned}
\gamma_{2 n}(1) & =(2 n+1) Q 0(2 n, 0) \\
& =(2 n+1)\left(\begin{array}{c}
2 n \\
n
\end{array}\right)\left(\frac{1}{2}\right)^{2 n}=(2 n+1) \frac{(2 n) !}{2^{2 n} n !^{2}} .
\end{aligned}
$$

In the case of large $n$, we can use the Stirling formula $n \approx n^{n} e^{-n} \sqrt{2 \pi n}$ and obtain $Q 0(2 n, 0) \approx 1 / \sqrt{\pi n}$ and $\gamma_{2 n}(1) \approx \sqrt{4 n / \pi}$. So we have got a closed form for the sum (2.18) of the return probabilities. The results are finite even in the case of $x=1$ (or $v=c$ ) because we assumed only a finite number $2 n$ of steps. Obviously, this assumption is adequate for all natural processes with finite duration.

Comment. The model of a one-dimensional random walk has only limited validity. Extensive considerations should take into account the interactions between different reference systems and changes of the observer's reference system. Up to now, we do not know enough about the exact ways of information between different reference systems and the long-term relation of their proper time. (Example: squared values like $\gamma_{2 n}(1)^{2} \approx$ $4 n / \pi$ or $\zeta_{2 n}(1)^{2} \approx 1 / \pi n$ (cf. (C.5)) can appear because of bidirectional information exchange during observation. We have seen in Section 1 that the familiar macroscopic geometrical appearance is not a primary thing, it is only a consequence of a discrete law. The above considerations suggest an information-theoretic approach to this law.)

Further research, especially combinatorial and graph-theoretic research (considering, e.g., branching loops), is necessary. The appendix demonstrates an example for possible connections of multiple random walks and [30] contains an example which starts out from the vacuum Maxwell equations.

\section{Appendix}

We now introduce the absorbing barriers which are drains and can be sources of new random walks with steps in another orthogonal direction. Then we show that in the case of an absorbing barrier in the origin after the start of the walk (and, otherwise, under the same basic conditions as in Theorem 2.1), the probability of nonabsorption is equivalent to $1 / \gamma(x)=\sqrt{1-x^{2}}$. At last, we investigate finite symmetric random walks with absorbing barriers.

A. Absorbing barriers. A Bernoulli random walk can have absorbing barriers. If there is an absorbing barrier at a point $a$ and the walking particle reaches it, the particle is absorbed. So the point $a$ is only a drain but is not a (direct) source for further walks within the same dimension (it can be a source of a walk in another dimension). We can get the resulting probability distribution by the subtraction of a "shifted" distribution from (2.10): we assume an absorbing barrier at $a>0$. We define

$$
P_{a}(n, k, p):=Q 0 P(n, k, p)-\left(\frac{p}{1-p}\right)^{a} Q 0 P(n, k-2 a, p)
$$


from which

$$
P_{a}(n+1, k, p)=p P_{a}(n, k-1, p)+(1-p) P_{a}(n, k+1, p)
$$

follows, that is, the inductive law (2.9) of a Bernoulli random walk holds. Additionally, the boundary condition $P_{a}(n, a, p)=0$ is fulfilled so that the point $a$ is only a drain but not a source. Therefore, $P_{a}(n, k, p)$ represents in case $k \leq a$ the probability that the particle passes point $k$ and continues moving. The points $k>a$ are not within reach for the particle starting at $k<a$.

In case $k>a, P_{a}(n, k, p)$ is negative. In the case of a simultaneous walk of two particles with starting points 0 and $2 a$, in which the particle starting at $2 a$ is the annihilating counterpart of the other starting at 0 , for $k>a$, the absolute value $\left|P_{a}(n, k, p)\right|$ can be interpreted as probability that the annihilating counterpart passes point $k$ if both particles make simultaneous steps in opposite directions. If this is not guaranteed, there is a chance that a particle passes the barrier.

RANDOM WALK WITH DELAYED ABSORBING BARRIER AT $k=0$. The starting coordinate $k=0$ plays a special role and it is reasonable to assume an absorbing barrier there also because of symmetry. But if this barrier is active from the beginning, the particle is absorbed at once so that the walk cannot begin and (A.1) has the meaningless result $P_{0}(n, k, p)=Q 0 P(n, k, p)-Q 0 P(n, k, p)=0$. However, if there is an absorption at $k=0$ after the walk has already started, we get a distribution which is worth further consideration. So we assume a delayed absorbing barrier at $k=0$ which is activated after the completion of the first step of the walk. The resulting probability distribution is given by the absolute values of

$$
Q 1 P(n, k, p):=(1-p) Q 0 P(n-1, k+1, p)-p Q 0 P(n-1, k-1, p)
$$

which is a modification of (A.1) because $Q 1 P(n, k, p)=(1-p) P_{1}(n-1, k+1, p)$. An absorbing barrier at $a=1$ is within reach and therefore active only from the second step on. The function $Q 1 P(n, k, p)$ is so defined that its symmetry center $k=0$ lies in this barrier. It fulfills the boundary conditions

$$
|Q 1 P(0,0, p)|=1, \quad Q 1 P(2 n, 0, p)=0 \quad \text { for } n \geq 1
$$

and the same inductive law as $Q 0 P$ in (2.9). For $n \geq 1$, a more compact form of $Q 1 P(n, k$, $p)$ is

$$
Q 1 P(n, k, p)=\frac{-k}{n} Q 0 P(n, k, p)
$$


because

$$
\begin{aligned}
Q 1 P & (n, k, p) \\
& =(1-p) Q 0 P(n-1, k+1, p)-p Q 0 P(n-1, k-1, p) \\
& =\frac{p^{(n+k) / 2}(1-p)^{(n-k) / 2}(n-1) !}{((n+k) / 2) !((n-k) / 2-1) !}-\frac{p^{(n+k) / 2}(1-p)^{(n-k) / 2}(n-1) !}{((n+k) / 2-1) !((n-k) / 2) !} \\
& =\left(\frac{n-k}{2 n}\right) \frac{p^{(n+k) / 2}(1-p)^{(n-k) / 2} n !}{((n+k) / 2) !((n-k) / 2) !}-\left(\frac{n+k}{2 n}\right) \frac{p^{(n+k) / 2}(1-p)^{(n-k) / 2} n !}{((n+k) / 2) !((n-k) / 2) !} \\
& =\left(\frac{-k}{n}\right) \frac{p^{(n+k) / 2}(1-p)^{(n-k) / 2} n !}{((n+k) / 2) !((n-k) / 2) !}=\frac{-k}{n} Q 0 P(n, k, p) .
\end{aligned}
$$

PAST DIFFERENCES. Equation (A.3) has a similarity to a finite difference along $k$. It represents the probability difference of the two ways coming from past. Therefore, we will call the accompanying operator past difference and use the symbol $\hat{\Delta}$ for it. If $\psi$ is a function of the variables $n, k$, and $p$, defined at least at $(n-1, k+1, p)$ and $(n-1, k-1, p)$, its past difference is

$$
\hat{\Delta} \psi(n, k, p)=(1-p) \psi(n-1, k+1, p)-p \psi(n-1, k-1, p) .
$$

Similar to the usual finite differences, we can form higher-order past differences; for example, the second-order past difference

$$
\begin{aligned}
Q 2 P(n, k, p):= & \hat{\Delta}^{2} Q O P(n, k, p)=\hat{\Delta} \hat{\Delta} Q O P(n, k, p) \\
= & (1-p) \hat{\Delta} Q 0 P(n-1, k+1, p)-p \hat{\Delta} Q 0 P(n-1, k-1, p) \\
= & (1-p)^{2} Q O P(n-2, k+2, p)+p^{2} Q O P(n-2, k-2, p) \\
& -2 p(1-p) Q O P(n-2, k, p) .
\end{aligned}
$$

For $n \geq 2$, we obtain

$$
\begin{array}{rl}
\hat{\Delta}^{2} Q & 0 P(n, k, p) \\
= & \hat{\Delta} \hat{\Delta} Q 0 P(n, k, p) \\
= & \hat{\Delta} Q 1 P(n, k, p)=\hat{\Delta}\left(\frac{-k}{n} Q 0 P(n, k, p)\right) \\
= & (1-p)\left(\frac{-k-1}{n-1}\right) Q 0 P(n-1, k+1, p)-p\left(\frac{1-k}{n-1}\right) Q 0 P(n-1, k-1, p) \\
= & \left(\frac{-k-1}{n-1}\right)((1-p) Q O P(n-1, k+1, p)-p Q 0 P(n-1, k-1, p)) \\
& -p\left(\frac{2}{n-1}\right) Q 0 P(n-1, k-1, p)
\end{array}
$$




$$
\begin{aligned}
& =\left(\frac{-k-1}{n-1}\right) Q 1 P(n, k, p)-\left(\frac{2}{n-1}\right)\left(\frac{n+k}{2 n}\right) Q 0 P(n, k, p) \\
& =\left(\frac{k(k+1)}{n(n-1)}\right) Q 0 P(n, k, p)-\left(\frac{n+k}{n(n-1)}\right) Q O P(n, k, p) \\
& =\frac{k^{2}-n}{n(n-1)} \operatorname{OP}(n, k, p) .
\end{aligned}
$$

The central second-order past differences

$$
Q 2 P(2 n, 0, p)=\frac{-1}{2 n-1} Q 0 P(2 n, 0, p)=\frac{-1}{2 n-1}\left(\begin{array}{c}
2 n \\
n
\end{array}\right)(1-p)^{n} p^{n}
$$

have a special meaning: because of

$$
\begin{aligned}
|Q 2 P(2 n, 0, p)| & =|\hat{\Delta} Q 1 P(2 n, 0, p)| \\
& =|(1-p) Q 1 P(2 n-1,1, p)-p Q 1 P(2 n-1,-1, p)| \\
& =(1-p)|Q 1 P(2 n-1,1, p)|+p|Q 1 P(2 n-1,-1, p)|,
\end{aligned}
$$

the absolute values

$$
|Q 2 P(2 n, 0, p)|=\frac{Q 0 P(2 n, 0, p)}{2 n-1}
$$

correspond for $n \geq 1$ to the probability of absorption after the $2 n$th step of the random walk specified in Appendix A.

Because in important physical equations (e.g., Schrödinger equation) the second derivative along location is related to the first derivative along time, it is worth mentioning that the second-order past difference (along $k$ ) is equivalent to a weighted first-order difference along $n$ :

$$
Q 2 P(n, k, p)=Q 0 P(n, k, p)-4 p(1-p) Q 0 P(n-2, k, p) .
$$

This follows from (A.8) and

$$
\begin{aligned}
Q O P(n, k, p)= & (1-p)^{2} Q O P(n-2, k+2, p)+p^{2} Q O P(n-2, k-2, p) \\
& +2 p(1-p) Q O P(n-2, k, p)
\end{aligned}
$$

B. The power series of $1 / \gamma(x)=\sqrt{1-x^{2}}$. Just like in Section 2.2.2, we now consider the power series of

$$
\zeta:[-1,1] \rightarrow \mathbb{R}, \quad \zeta(x)=\sqrt{1-x^{2}}
$$


So, $\zeta(x)=1 / \gamma(x)$ for $|x|<1$ and $\zeta(-1)=\zeta(1)=0$. Because of $\sqrt{1+z}=f_{1 / 2}(z)$, we get analogously to (2.7),

$$
\begin{aligned}
\sqrt{1+z}= & \sum_{l=0}^{\infty}\left(\begin{array}{c}
\frac{1}{2} \\
l
\end{array}\right) z^{l} \\
= & 1+\frac{1 / 2}{1} z^{1}+\frac{(1 / 2) \cdot(-1 / 2)}{1 \cdot 2} z^{2}+\frac{(1 / 2) \cdot(-1 / 2) \cdot(-3 / 2)}{1 \cdot 2 \cdot 3} z^{3} \\
& +\frac{(1 / 2) \cdot(-1 / 2) \cdot(-3 / 2) \cdot(-5 / 2)}{1 \cdot 2 \cdot 3 \cdot 4} z^{4}+\cdots \\
= & 1+\frac{1}{2^{1} \cdot 1 !} z^{1}-\frac{1 \cdot 1}{2^{2} \cdot 2 !} z^{2}+\frac{1 \cdot 1 \cdot 3}{2^{3} \cdot 3 !} z^{3}-\frac{1 \cdot 1 \cdot 3 \cdot 5}{2^{4} \cdot 4 !} z^{4}+\cdots \\
= & 1-\sum_{l=1}^{\infty} \frac{1}{2 l-1}\left(\begin{array}{c}
2 l \\
l
\end{array}\right)\left(\frac{-z}{4}\right)^{l}
\end{aligned}
$$

from which

$$
\zeta(x)=\sqrt{1-x^{2}}=1-\sum_{l=1}^{\infty} \frac{1}{2 l-1}\left(\begin{array}{c}
2 l \\
l
\end{array}\right)\left(\frac{x}{2}\right)^{2 l}
$$

follows. So, in case $4 p(1-p)=x^{2}$, we obtain, by (2.12), (2.15), and (A.10),

$$
\begin{aligned}
\zeta(x) & =\sqrt{1-x^{2}}=1-\sum_{n=1}^{\infty} \frac{1}{2 n-1} Q O P(2 n, 0, p) \\
& =1+\sum_{n=1}^{\infty} Q 2 P(2 n, 0, p)=1-\sum_{n=1}^{\infty}|Q 2 P(2 n, 0, p)|
\end{aligned}
$$

Because $|Q 2 P(2 n, 0, p)|$ is the probability of absorption after the $2 n$th step, $\sum_{n=1}^{\infty}|Q 2 P(2 n, 0, p)|$ is the total probability of absorption. Therefore, $\sqrt{1-x^{2}}$ is the probability of nonabsorption, respectively, nonreturn (of “escape"). We summarize this in the following theorem.

THEOREM B.1. If a particle makes a Bernoulli random walk, in which each step is directed from point $k$ to $k+1$ with probability $p$ and from point $k$ to $k-1$ with probability $1-p$, and the particle is absorbed if it returns to the starting point, and $x^{2}=4 p(1-p)$, then the probability of nonabsorption (of "escape") is $\zeta(x)=\sqrt{1-x^{2}}$.

REMARK B.2. More concrete formulations of this theorem are possible. Due to experimental results, we know that the energy of a photon can be distributed. If $E$ is the energy of the photon, its frequency $v$ is given by $v=E / h$, in which $h$ is Planck's constant ( $h \approx 6.626 \cdot 10^{-34} \mathrm{Js}$ ). At this, a reduction of the photon's frequency is equivalent to a dilation of its time period. So, we can regard the energy of a received photon as the nonreturning (escaping) part of its initial energy and we can state the following theorem. 
THEOREM B.3. Let $\gamma(x)=1 / \sqrt{1-x^{2}}$ represent the (approximative) time dilation factor of reference system $A$ relative to reference system $B$ as in Theorem 2.1. If a photon is emitted in $B$ with energy $E_{e}=h v_{e}$ and is absorbed in $A$, the maximal absorption energy $E_{a}=h v_{a}$ in system $A$ is given by $E_{a}=E_{e} \sqrt{1-x^{2}}$. So the quotient $E_{a} / E_{e}=v_{a} / v_{e}$ (i.e., the part of the photon's energy which can escape and arrive in $A$ in comparison with initial energy of the photon) is (approximatively) equivalent to the probability (i.e., the expectation value of the frequency of nonreturning (escaping) walks in comparison with the total frequency or total number of walks) that there is no return to the starting point during a Bernoulli random walk, in which each step is directed from point $k$ to $k+1$ with probability $p$ and from point $k$ to $k-1$ with probability $1-p$, where $4 p(1-p)=x^{2}$.

\section{Case $x=1$, respectively, $v=c$, with absorbing barrier}

SYMmETRY. In Section 2.2.4, we have seen that in case $x=1$, respectively $v=c$, a symmetric random walk results. In case $x=1$ or $v=c$, also the random walk with absorbing barrier, described in Appendix A, becomes symmetric, because $p=1-p=$ $1 / 2$ with (2.15) and the barrier (which is active from the second step on) is located in the starting point $k=0$. The probability that after the $n$th step the point $k$ is reached and the walk continues is given by the absolute value of

$$
Q 1(n, k):=Q 1 P\left(n, k, \frac{1}{2}\right)
$$

The first values of $Q 1(n, k)$ are shown in Table C.1.

FINITE RANDOM WALK. By Appendix B, in case $x=1$, the probability of absorption (or return to the starting point) is 1 if the number of steps in the walk has no upper limit. Because in physical reality within finite time only a finite number of steps are possible, we consider the finite partial sums

$$
\zeta_{2 n}(x):=1+\sum_{m=1}^{n} Q 2 P\left(2 m, 0, \frac{1+\sqrt{1-x^{2}}}{2}\right)
$$

of the power series of $\zeta(x)$. Similarly, as in Section 2.2.4 for $\gamma_{2 n}(1)$, we can find a closed form for $\zeta_{2 n}(1)$. For $n>0$, we get, by (A.13),

$$
Q O P\left(n-2,0, \frac{1}{2}\right)+Q 2 P\left(n, 0, \frac{1}{2}\right)=Q O P\left(n, 0, \frac{1}{2}\right)
$$

so that with $Q 0 P(0,0,1 / 2)=1$, by induction, it follows that

$$
\zeta_{2 n}(1)=1+\sum_{m=1}^{n} Q 2 P\left(2 m, 0, \frac{1}{2}\right)=Q 0 P\left(2 n, 0, \frac{1}{2}\right)=\frac{(2 n) !}{2^{2 n}(n !)^{2}} .
$$

In the case of large $n$, we can use the Stirling formula to obtain

$$
\zeta_{2 n}(1) \approx \frac{1}{\sqrt{\pi n}}
$$


TABLE C.1. The first values of $Q 1(n, k)=Q 1(n, k, 1 / 2)$. The absolute value of the number in row $n$ and column $k$ represents the number of ways which lead without absorption to point $k$ after the $n$th steps. Multiplication of the number by the factor $2^{-n}$ yields $Q 1(n, k)$. The last column contains these factors. Multiplication of the underlined number in row $n$ by $2^{-n}$ yields $Q 1(2 n-$ $1,-1)=|Q 1(2 n-1,-1)-Q 1(2 n-1,-1)| / 2=|Q 2 P(2 n, 0,1 / 2)|$ which is the probability of absorption after $2 n$ steps. It is visible that the numbers result from the addition of two Pascal triangles with opposite sign, one starting at $(n, k)=(1,-1)$ and the other starting at $(n, k)=(1,1)$, so that at $k=0$ annihilation occurs.

\begin{tabular}{|c|c|c|c|c|c|c|c|c|c|c|c|c|c|c|}
\hline & \multicolumn{14}{|c|}{$k$} \\
\hline & -6 & -5 & -4 & -3 & -2 & -1 & 0 & 1 & 2 & 3 & 4 & 5 & 6 & \\
\hline 1 & & & & & & $\underline{1}$ & & -1 & & & & & & $\cdot 2^{-1}$ \\
\hline 2 & & & & & 1 & & 0 & & -1 & & & & & $\cdot 2^{-2}$ \\
\hline 3 & & & & 1 & & $\underline{1}$ & & -1 & & -1 & & & & $\cdot 2^{-3}$ \\
\hline 4 & & & 1 & & 2 & & 0 & & -2 & & -1 & & & $\cdot 2^{-4}$ \\
\hline 5 & & 1 & & 3 & & $\underline{2}$ & & -2 & & -3 & & -1 & & $\cdot 2^{-5}$ \\
\hline 6 & 1 & & 4 & & 5 & & 0 & & -5 & & -4 & & -1 & $\cdot 2^{-6}$ \\
\hline
\end{tabular}

where $1-\zeta_{2 n}(1)$ is the probability of absorption in case $x=1$ or $p=1-p=1 / 2$ when making at most $2 n$ steps. The probability of absorption (A.12) after the $2 n$th step is given by the negative second-order past difference along $k$ :

$$
-Q 2 P\left(2 n, 0, \frac{1}{2}\right)=-\hat{\Delta}^{2} Q O P\left(2 n, 0, \frac{1}{2}\right)=\frac{1}{2 n-1} Q O P\left(2 n, 0, \frac{1}{2}\right) \approx \frac{1}{\sqrt{4 \pi n^{3}}},
$$

and because of the Schrödinger equation, it is remarkable that, with (A.13), this is equivalent to the negative (first-order) finite difference along $n$ :

$$
-Q 2 P\left(2 n, 0, \frac{1}{2}\right)=Q O P\left(2 n-2,0, \frac{1}{2}\right)-Q 0 P\left(2 n, 0, \frac{1}{2}\right)
$$

We have seen that the discrete differentiation, which is defined in Appendix A, leads to a probability distribution with absorbing barrier. Separation (and distinction) of the ways on both sides of the barrier is connected with this. We should recall that, in physical experiments (e.g., double-slit experiment), such separation is also connected with absorption (and emission) of photons at systems with rest mass.

\section{REFERENCES}

[1] M. Bauer, C. Godrèche, and J. M. Luck, Statistics of persistent events in the binomial random walk: will the drunken sailor hit the sober man? J. Statist. Phys. 96 (1999), no. 5-6, 963-1019.

[2] W. Bauer and S. Pratt, Size matters: origin of binomial scaling in nuclear fragmentation experiments, Phys. Rev. C 59 (1999), 2695-2698. 
[3] S. Bottini, New polynomial law of hadron mass, preprint, 1998, http://arxiv.org/abs/ hep-ph/9810405.

[4] D. S. Bridges, Constructive Functional Analysis, Research Notes in Mathematics, vol. 28, Pitman, Massachusetts, 1979.

[5] L. Brillouin, Science and Information Theory, 2nd ed., Academic Press, New York, 1962.

[6] W. Feller, An Introduction to Probability Theory and Its Applications. Vol. I, John Wiley \& Sons, New York, 1957.

[7] _ An Introduction to Probability Theory and Its Applications. Vol. II, 2nd ed., John Wiley \& Sons, New York, 1971.

[8] A. A. Fraenkel and Y. Bar-Hillel, Foundations of Set Theory, Studies in Logic and the Foundations of Mathematics, North-Holland Publishing, Amsterdam, 1958.

[9] H.-C. Fu and R. Sasaki, Negative binomial states of quantized radiation fields, J. Phys. Soc. Japan 66 (1997), no. 7, 1989-1994.

[10] A. Giovannini, S. Lupia, and R. Ugoccioni, The negative binomial distribution in quark jets with fixed flavour, Phys. Lett. B 388 (1996), no. 3, 639-647.

[11] S. Gottwald, H. Kästner, and H. Rudolph (eds.), Meyers kleine Enzyklopädie Mathematik, 14th ed., Meyers Lexikonverlag, Mannheim, 1995.

[12] S. Hegyi, Multiplicity distributions in strong interactions: a generalized negative binomial model, Phys. Lett. B 387 (1996), no. 3, 642-650.

[13] A. Heyting, Intuitionism. An Introduction, 3rd revised ed., North-Holland Publishing, Amsterdam, 1971.

[14] D. Hilbert, Über das Unendliche, Math. Ann. 95 (1926), 161-190 (German).

[15] A. M. Jaglom and I. M. Jaglom, Wahrscheinlichkeit und Information [Probability and Information], 4th ed., VEB Deutscher Verlag der Wissenschaften, Berlin, 1984.

[16] A. Khrennikov, Ultrametric Hilbert space representation of quantum mechanics with a finite exactness, Found. Phys. 26 (1996), no. 8, 1033-1054.

[17] _ Non-Archimedean Analysis: Quantum Paradoxes, Dynamical Systems and Biological Models, Mathematics and Its Applications, vol. 427, Kluwer Academic Publishers, Dordrecht, 1997.

[18] A. Khrennikov and Y. Volovich, Discrete time leads to quantum-like interference of deterministic particles, Proc. Int. Conf. Quantum Theory: Reconsideration of Foundations, Ser. Math. Modelling in Phys., Engin., and Cogn. Sc., Växjö University Press, Växjö, 2002, pp. 441-454.

[19]___ Interference effect for probability distributions of deterministic particles, Proc. Int. Conf. Quantum Theory: Reconsideration of Foundations, Ser. Math. Modelling in Phys., Engin., and Cogn. Sc., Växjö University Press, Växjö, 2002, pp. 455-462.

[20] K. Knopp, Theorie und Anwendung der unendlichen Reihen, Die Grundlehren der mathematischen Wissen schen Wissenschaften, vol. 2, Springer-Verlag, Berlin, 1964.

[21] H. W. Lee and L. S. Levitov, Estimate of minimal noise in a quantum conductor, preprint, 1995, http://arxiv.org/abs/cond-mat/9507011.

[22] L. S. Levitov, H. Lee, and G. B. Lesovik, Electron counting statistics and coherent states of electric current, J. Math. Phys. 37 (1996), no. 10, 4845-4866.

[23] C. MacLaurin, A Treatise of Fluxions, Vol. 1-2, T. W. \& T. Ruddimans, Edinburgh, 1742.

[24] A. I. Markushevich, Theory of Functions of a Complex Variable. Vol. I, Chelsea Publishing, New York, 1977.

[25] S. G. Matinyan and E. B. Prokhorenko, Branching processes and multiparticle production, Phys. Rev. D 48 (1993), no. 11, 5127-5132.

[26] A. Messiah, Quantenmechanik. Band 1 [Quantum Mechanics. Vol. 1], 2nd ed., Walter de Gruyter, Berlin, 1991.

[27] N. Nakajima, M. Biyajima, and N. Suzuki, Analysis of cumulant moments in high energy hadron-hadron collisions through truncated multiplicity distributions, Phys. Rev. D 54 (1996), no. 7, 4333-4336. 
[28] P. O'Hara, Clebsch-Gordan coefficients and the binomial distribution, preprint, 2001, http://arxiv.org/abs/quant-ph/0112096.

[29] W. Orthuber, A discrete and finite approach to past proper time, preprint, 2002, http://arxiv.org/abs/quant-ph/0207045.

[30] _ A discrete approach to the vacuum Maxwell equations and the fine structure constant, preprint, 2003, http://arxiv.org/abs/quant-ph/0312188.

[31] _ To the finite information content of the physically existing reality, preprint, 2001, http://arxiv.org/abs/quant-ph/0108121.

[32] I. A. Poletajew, Kybernetik, 3rd ed., VEB Deutscher Verlag der Wissenschaften, Berlin, 1964.

[33] R. R. Puri, S. Arun Kumar, and R. K. Bullough, Stroboscopic theory of atomic statistics in the micromaser, preprint, 1999, http://arxiv.org/abs/quant-ph/9910103.

[34] F. Spitzer, Principles of Random Walks, 2nd ed., Graduate Texts in Mathematics, vol. 34, Springer-Verlag, New York, 1976.

[35] O. G. Tchikilev, Multiplicity distributions in $e^{+} e^{-}$annihilation into hadrons and pure birth branching processes, Phys. Lett. B 471 (2000), no. 4, 400-405.

[36] A. S. Troelstra and D. van Dalen, Constructivism in Mathematics. An Introduction. Vol. I, North-Holland Publishing, Amsterdam, 1988.

[37] C Constructivism in Mathematics. An Introduction, Vol. II, North-Holland Publishing, Amsterdam, 1988.

[38] H. Weyl, Das Kontinuum. Kritische Untersuchungen über die Grundlagen der Analysis, Veit \& Co., Leipzig, 1918.

[39]__ Über die neue Grundlagenkrise der Mathematik, Math. Z. 10 (1921), 39-79 (German).

[40]___ Randbemerkungen zu Hauptproblemen der Mathematik, Math. Z. 20 (1924), 131150.

Wolfgang Orthuber: Klinik für Kieferorthopädie der Universität Kiel, Arnold-Heller Street 16, 24105 Kiel, Germany

E-mail address: orthuber@kfo-zmk.uni-kie1.de 


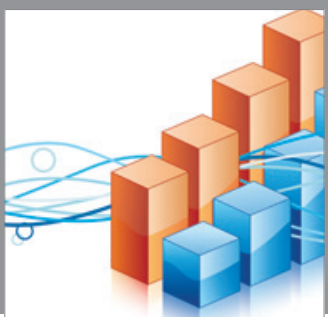

Advances in

Operations Research

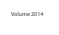

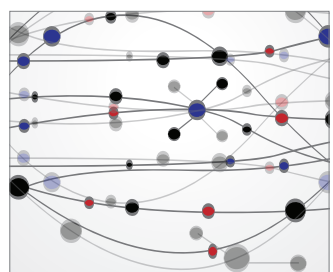

\section{The Scientific} World Journal
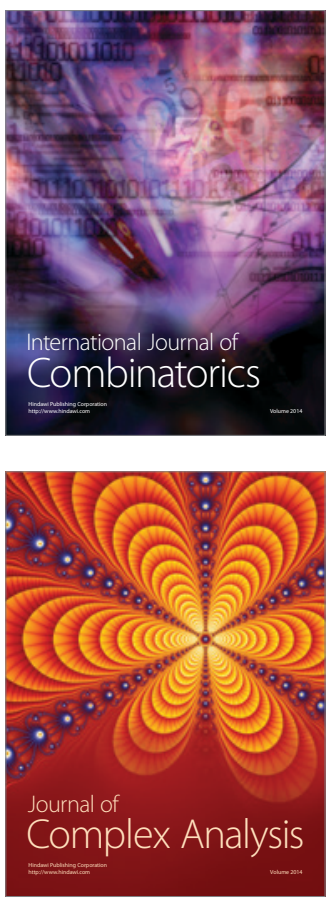

International Journal of

Mathematics and

Mathematical

Sciences
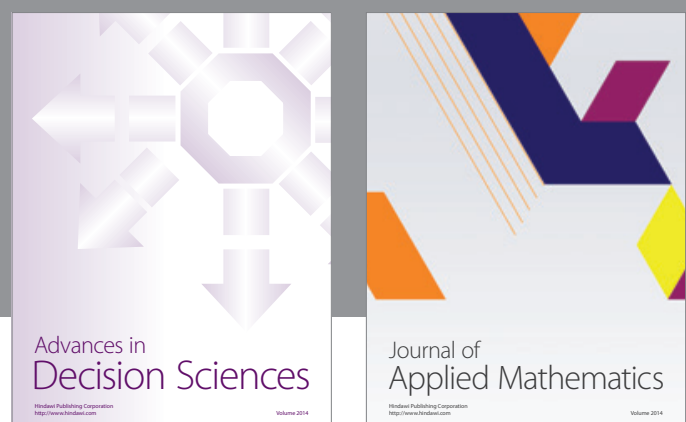

Journal of

Applied Mathematics
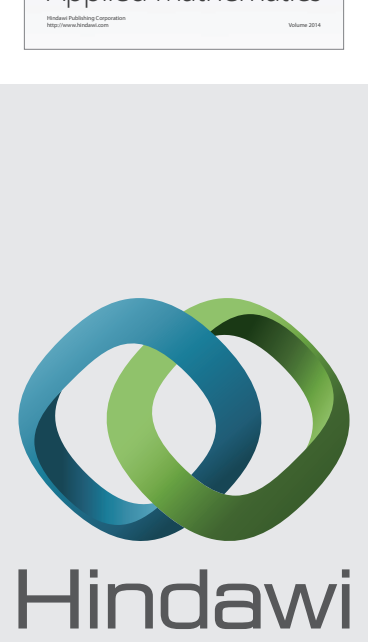

Submit your manuscripts at http://www.hindawi.com
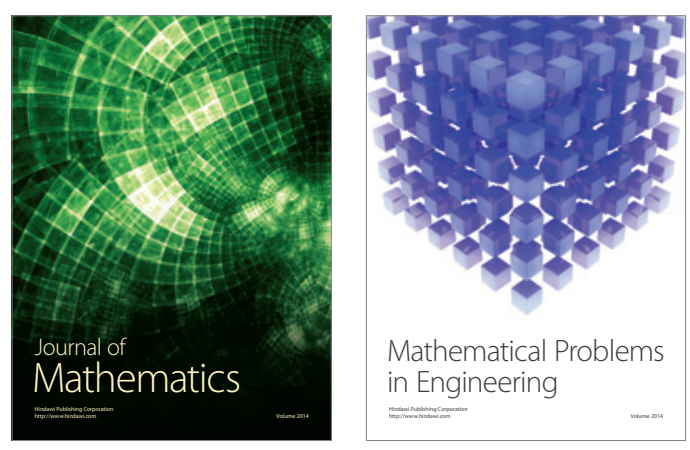

Mathematical Problems in Engineering
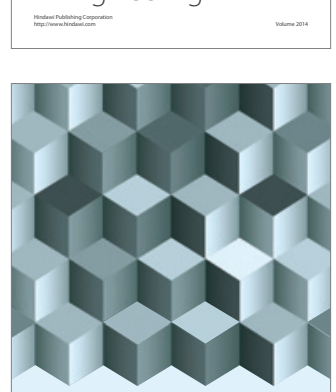

Journal of

Function Spaces
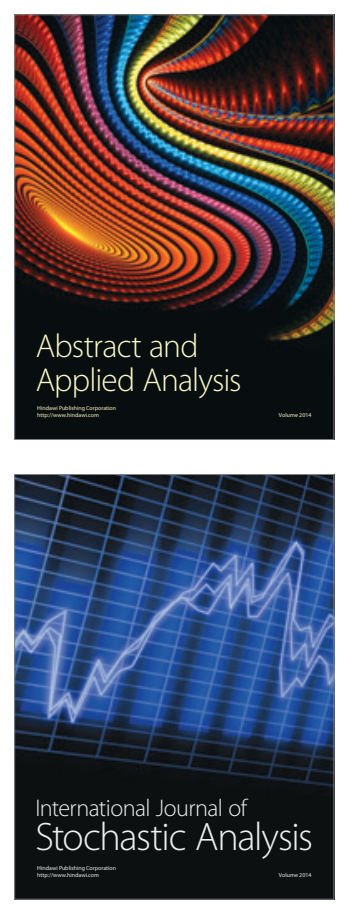

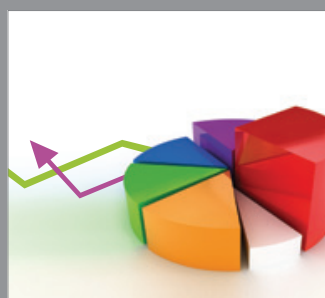

ournal of

Probability and Statistics

Promensencen
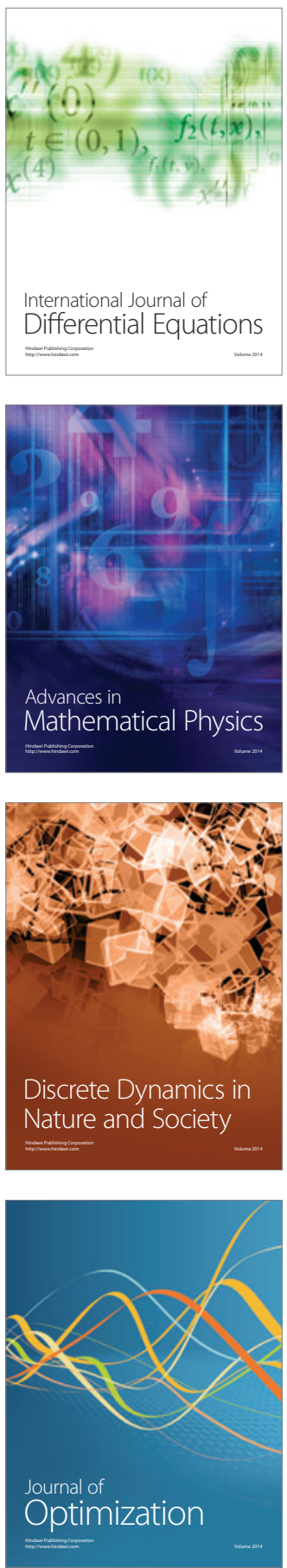